\title{
LINEAR POLARIZATION PARALLEL PLATE SLOT ANTENNA
}

\author{
Manuel Sierra Castañer , JoaquínIzquierdo ,Manuel Sierra Pérez , José Luis \\ Fernández Jambrina , María Vera Isasa
}

\section{ABSTRACT}

We show here the design of a linearly polarised flat antenna. The main goal is getting a low cost antenna to use for the reception of Hispasat satellite emissions in the 12 $\mathrm{GHz}$ band. The gain needed for the antenna is around $30 \mathrm{dBi}$. A rectangular array of slots in the upper side of a parallel plate wave guide form the main antenna structure. The guide is excited by one planar feeding structure consisting of an array of rectangular microstrip patches.

Parallel plate slot antennas have been widely studied by Ando and Hirokawa [1], from $12 \mathrm{GHz}$ to Millimeter Wave frequencies. We also modify the feeding network, the design procedure and the materials used to build of the radiation surface.

\section{PARAllel plate WAVE-GUIDE}

The antenna main structure is made with two metallic plates, and filled by two kind of dielectric materials: a low density foam dielectric and an epoxy fibre glass. The ground plane is a $3 \mathrm{~mm}$ aluminium plate and the upper plate is a copper plated, low thickness fiberglass sheet. The upper plate will support the slots printed antenna, while the ground plane supports the printed feeding structure at both sides of the antenna. Fig. 1 shows a schematic section if the antenna structure.

The shape of the antenna is square of $36 \mathrm{~cm} \times 36 \mathrm{~cm}$, with the slots printed in the upper plate, all of them parallel to the others to give linear polarisation. To get a plane feeding wave inside the guide an array of rectangular patches were placed in the side of the antenna and fed with equal amplitude and phase. Fig. 2 shows a schematic drawing of the antenna and
antenna.

\section{FEEDING STRUCTURE AND PLANE WAVE EXCITATION}

The feeding network has been printed in a $0.254 \mathrm{~mm}$ thick teflon-glass substrate. It is based on an array of rectangular resonant patches fed by a microstrip line to get an homogeneous field in a plane wave inside the guide. The total number of patches is 24 . With this number the ripple of the field inside the guide (amplitude and phase) is really small. The feeding structure is a parallel feeding network printed in the same substrate as the resonant patches and made with a set of two and three way power dividers and quarter wavelength transformers.

\section{SLOT COLUMNS}

The radiating structure is formed by columns of slots printed in the upper plate. Each column acts as a full radiating element in a travelling wave antenna, and we can associate to it a radiated power and a set of transmission and reflection parameters in the waveguide. The radiated power depends on the slot length, that is constant for all the slots in a column, and we can adjust this length to control the excitation along the antenna 
aperture. The reflected power inside the parallel plate waveguide must be very low, but as the radiated power is increased, the reflection also increases.

To reduce the reflection from the slots, the radiating element consists on a set of three slots. The central one is longer than the side ones and it radiates most of the power. The other two slots are much shorter than the resonance length and are placed to reduce the reflection of the main slot. The structure to be analysed is showed in figure 3 . There are two regions, the half free space and the waveguide. For the half free space we consider periodicity in both directions, to consider the coupling among all the slots. For the inner waveguide we consider the periodicity in one direction (the direction parallel to the slots). The upper plate is considered zero thickness. The integral system of equations (1) is solved by the Galerkin Method of Moments, and the equivalent magnetic currents on each of the three slots are obtained.

For $\mathrm{j}=1: 3, \quad \quad \vec{H}_{i n}+\sum_{i} \vec{M}_{i}\left(\vec{r}_{i}\right) \otimes \overline{\bar{G}}^{W G}\left(\vec{r}_{j} / \vec{r}_{i}\right)=-\sum_{i} \vec{M}_{i}\left(\vec{r}_{i}\right) \otimes \overline{\bar{G}}^{H S}\left(\vec{r}_{j} / \vec{r}_{i}\right)$

The base functions are complete sinusoidal ones for each slot (2). One base function is enough for solving the system [2].

$$
\bar{m}_{i}(\vec{r})=\frac{1}{w} \sin \frac{k \pi}{l}\left(z+\frac{l}{2}\right) \hat{z} \quad k=1 \cdots N
$$

For the analysis of the inner waveguide hybrid $\mathrm{TE}^{y}$ and $\mathrm{TM}^{\mathrm{y}}$ modes are used. For a two dielectrics geometry the wavenumber for each direction are [3]:

TE ${ }^{y}$ modes:

$$
\frac{k_{y_{1}}}{\mu_{1}} \cdot \cot \left[k_{y_{1}}, h_{l}\right]=-\frac{k_{y_{2}}}{\mu_{2}} \cdot \cot \left[k_{y_{2}}, h_{2}\right]
$$

TM ${ }^{\mathrm{y}}$ modes:

$$
\frac{k_{y_{1}}}{\varepsilon_{1}} \cdot \tan \left[k_{y_{1}} \cdot h_{l}\right]=-\frac{k_{y_{2}}}{\varepsilon_{2}} \cdot \tan \left[k_{y_{2}} \cdot h_{2}\right]
$$

These expresions are completed with the wavenumber in a periodic structure,

$$
\begin{aligned}
& k_{x}{ }^{2}+k_{y_{1}}{ }^{2}+k_{z}{ }^{2}=\omega^{2} \mu_{1} \varepsilon_{I} \\
& k_{x}{ }^{2}+k_{y_{z}}{ }^{2}+k_{z}{ }^{2}=\omega^{2} \mu_{2} \varepsilon_{2} \\
& k x=k_{0} \cdot\left(\frac{2 m \pi}{a}+\sin \theta \cos \phi\right)
\end{aligned}
$$

The length of the reflection cancelling slots is calculated to minimize the reflection coefficient. Then the radiated power is calculated for each structure. This power depends mainly on the length of the slots, and less on the distance between slots. Figure 4 shows the length of side slots as a function of the main slot length, and figure 5 shows the radiated power (\% over incident power) as a function of the length of main slot.

\section{DESIGN OF THE RADIATION SURFACE}

The length of each slot in one line is calculated to have uniform amplitude in the antenna aperture. The radiated power by one line of slots depends on its length. This set of three slots generates a change in the phase of the transmission field inside the guide and aiso the phase of the radiated field changes from the phase of the field inside the waveguide. The line of slots must be placed in theory one wavelength apart measured inside the guide to get a broadside antenna, but considering that two changes of phase in the field, the distance between each line of slots must be reduced. 


\section{CONCLUSIONS}

A design method for the synthesis linearly polarised parallel plate antenna has been presented. This work pretends to study the possibilities of this kind of antenna in a two dielectric stmicture. Also, a printed feeding structure for the parallel plate waveguide has been included to avoid rectangular guide slotted feeding network, that made the antenna more thick and expensive. Measurements will be showed at the conference. This work is included into the project FEDER $n^{\circ} 1-F D 97-0276-\mathrm{C02}-02$. We want to thank the financial supporting from FEDER

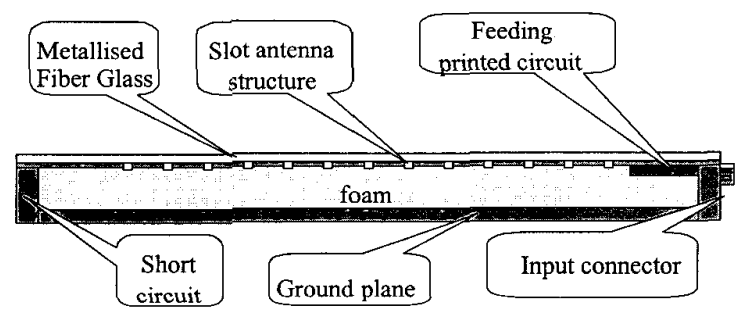

Fig. 1. Scheme of the transmission line section.

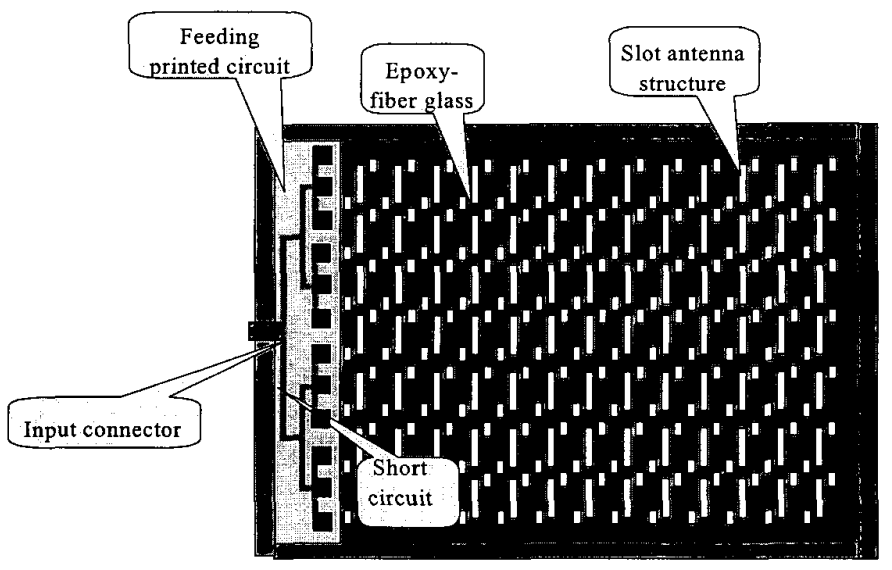

Fig. 2. Scheme of the antenna structure. 


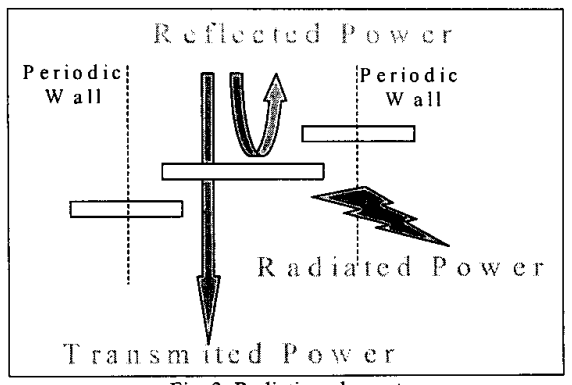

Fig. 3. Radiating element

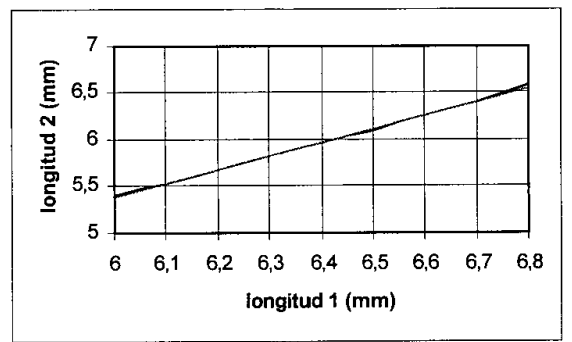

Fig. 4: Length of side slots

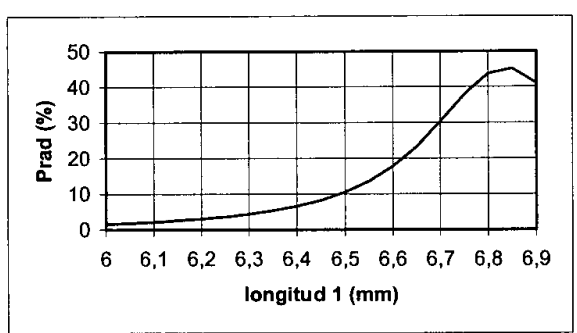

Fig. 5: Radiated Power

\section{References}

[1] J. Hirokawa, M. Ando. "Single-Layer Feed Waveguide Consisting of Posts for Plane TEM Wave Excitation in Parallel Plates". IEEE Trans. on Antennas and Propagation. Vol. 45, No.5 Mayo 1998, pp. 625-630.

[2] Jiro Hirokawa, Makoto Ando, N. Goto. "Analysis of Slot Coupling in a Radial Line Slot Antenna for DBS reception" IEE Proceedings, Vol 137, Pt. H, No.5, October 1990. Pag. 249-254

[3] C.A. Balanis "Advanced Engineering Electromagnetics", John Wiley \& Sons", Nueva York, 1989. 